\title{
Relationship of nine constants
}

\section{Michael Snyder}

Department of Physics and Astronomy, University of Louisville, Louisville, USA; m0snyd04@1ouisville.edu

Received 25 June 2013; revised 25 July 2013; accepted 4 August 2013

Copyright (C) 2013 Michael Snyder. This is an open access article distributed under the Creative Commons Attribution License, which permits unrestricted use, distribution, and reproduction in any medium, provided the original work is properly cited.

\begin{abstract}
Through the process of trial and error, four unitless equations made up of nine constants have been found with exact answers. The related constants are the Speed of Light [1], the Planck constant [2], Wien's displacement constant [3], Avogadro's number [4], the universal Gravity constant [5], the Ampere constant [6], the Faraday constant [7], the Gas constant [8] and Apery's constant [9].
\end{abstract}

Keywords: Planck; Wien; Avogadro; Faraday; Apery; Ampere

\section{INTRODUCTION}

At the end of the spring semester 2013, I had found an expression of a few physical constants that gave the correct value of the universal Gravity constant [5]. I shared my findings with my classmates and they all pointed out the units were incorrect.

This started the search for a unitless expression of physical constants similar in form to the Fine Structure constant but with more constants.

In the context of this paper, the term unitless is defined as all the exponents of the units on the left hand side of the equation are equal to zero and the right hand side of the equation is represented by only a numeric expression.

\section{MAIN BODY}

The first few equations were found by trial and error. One would literally examine a listing of physical constants and guess which set of constants multiplied together and divided by another set of multiplied constants produces an answer with units raised to the zero power.

I had the limited success of finding the Fine Structure constant over and over again. At this point I changed my strategy by writing a program that would try every combination of a set of constants within a certain integer range of exponents, with its dimensionality equal to a selected SI unit. This strategy worked in the sense that it produced a large set of equations, of the selected constants that had the required SI units of seconds or meters, etc.

The programming process and the testing the programs happened over a few weeks and various sets of physical constants where tried. Overall the physical constants that produced the most equations were selected to be in the final set of nine presented in this paper.

A few things happened concurrently that allowed me to find the equations presented in this paper. One was that I started using a unit of an ampere-mole as a range extender in my search programs. The derived unit could be removed from the final answers yet its presence in the program allowed more equations to be found.

The second was that it occurred to me that the structure of the programs that I had written; could search for unitless equations too. The third was that I added the Faraday constant to the primary set of search constants. I intended to use the Faraday constant as a more robust replacement for the derived ampere-mole constant, and was hoping for similar results.

A few minutes later, the first of many unitless equations appeared on the screen. Through the process of trial and error I had found a set of eight physical constants that produced unitless equations.

Once a pattern was found in the first few equations, a new program in the Cuda GPU language was written to find unitless combinations expressed as the powers of the constants. A program listing is included for completeness as Appendix I.

A set of 200 unitless equations are shown in Table 1, and Eq.1 through Eq.4 are the results of the reduced row echelon form of Table 1. The reduced row echelon operation on Table 1, results with two rows.

Eq.1 represents the first row and Eq.2 represents the second row of the reduced row echelon form. Eq.3 represents the multiplication of the Eq.1 and Eq.2 and Eq.4 represents the quotient of Eq.1 and Eq. 2. One can 
Table 1. A family of unitless equations.

\begin{tabular}{|c|c|c|c|c|c|c|c|c|c|}
\hline Index Number & $\operatorname{Ln}\left(c^{0}\right.$ & $\mathbf{h}^{0}$ & $\mathrm{~A}^{0}$ & $\mathbf{R}^{0}$ & $\mathbf{w}^{0}$ & $\mathrm{G}^{0}$ & $\mathrm{~N}^{0}$ & $\left.F^{0}\right)=$ & value \\
\hline 1 & 0 & 0 & 0 & 0 & 0 & 0 & 0 & 0 & 0 \\
\hline 2 & -64 & -70 & -2 & 69 & 69 & -1 & -71 & 2 & 0.29664 \\
\hline 3 & -128 & -140 & -4 & 138 & 138 & -2 & -142 & 4 & 0.59328 \\
\hline 4 & 193 & 211 & 6 & -208 & -208 & 3 & 214 & -6 & 0.71252 \\
\hline 5 & -192 & -210 & -6 & 207 & 207 & -3 & -213 & 6 & 0.88992 \\
\hline 6 & 129 & 141 & 4 & -139 & -139 & 2 & 143 & -4 & 1.0092 \\
\hline 7 & 65 & 71 & 2 & -70 & -70 & 1 & 72 & -2 & 1.3058 \\
\hline 8 & 1 & 1 & 0 & -1 & -1 & 0 & 1 & 0 & 1.6024 \\
\hline 9 & -63 & -69 & -2 & 68 & 68 & -1 & -70 & 2 & 1.8991 \\
\hline 10 & -127 & -139 & -4 & 137 & 137 & -2 & -141 & 4 & 2.1957 \\
\hline 11 & 194 & 212 & 6 & -209 & -209 & 3 & 215 & -6 & 2.315 \\
\hline 12 & -191 & -209 & -6 & 206 & 206 & -3 & -212 & 6 & 2.4924 \\
\hline 13 & 130 & 142 & 4 & -140 & -140 & 2 & 144 & -4 & 2.6116 \\
\hline 14 & 66 & 72 & 2 & -71 & -71 & 1 & 73 & -2 & 2.9082 \\
\hline 15 & 2 & 2 & 0 & -2 & -2 & 0 & 2 & 0 & 3.2049 \\
\hline 16 & -62 & -68 & -2 & 67 & 67 & -1 & -69 & 2 & 3.5015 \\
\hline 17 & -126 & -138 & -4 & 136 & 136 & -2 & -140 & 4 & 3.7982 \\
\hline 18 & 195 & 213 & 6 & -210 & -210 & 3 & 216 & -6 & 3.9174 \\
\hline 19 & -190 & -208 & -6 & 205 & 205 & -3 & -211 & 6 & 4.0948 \\
\hline 20 & 131 & 143 & 4 & -141 & -141 & 2 & 145 & -4 & 4.214 \\
\hline 21 & 67 & 73 & 2 & -72 & -72 & 1 & 74 & -2 & 4.5107 \\
\hline 22 & 3 & 3 & 0 & -3 & -3 & 0 & 3 & 0 & 4.8073 \\
\hline 23 & -61 & -67 & -2 & 66 & 66 & -1 & -68 & 2 & 5.1039 \\
\hline 24 & -125 & -137 & -4 & 135 & 135 & -2 & -139 & 4 & 5.4006 \\
\hline 25 & 196 & 214 & 6 & -211 & -211 & 3 & 217 & -6 & 5.5198 \\
\hline 26 & -189 & -207 & -6 & 204 & 204 & -3 & -210 & 6 & 5.6972 \\
\hline 27 & 132 & 144 & 4 & -142 & -142 & 2 & 146 & -4 & 5.8165 \\
\hline 28 & 68 & 74 & 2 & -73 & -73 & 1 & 75 & -2 & 6.1131 \\
\hline 29 & 4 & 4 & 0 & -4 & -4 & 0 & 4 & 0 & 6.4097 \\
\hline 30 & -60 & -66 & -2 & 65 & 65 & -1 & -67 & 2 & 6.7064 \\
\hline 31 & -124 & -136 & -4 & 134 & 134 & -2 & -138 & 4 & 7.003 \\
\hline 32 & 197 & 215 & 6 & -212 & -212 & 3 & 218 & -6 & 7.1223 \\
\hline 33 & -188 & -206 & -6 & 203 & 203 & -3 & -209 & 6 & 7.2997 \\
\hline 34 & 133 & 145 & 4 & -143 & -143 & 2 & 147 & -4 & 7.4189 \\
\hline 35 & 69 & 75 & 2 & -74 & -74 & 1 & 76 & -2 & 7.7155 \\
\hline 36 & 5 & 5 & 0 & -5 & -5 & 0 & 5 & 0 & 8.0122 \\
\hline 37 & -59 & -65 & -2 & 64 & 64 & -1 & -66 & 2 & 8.3088 \\
\hline 38 & -123 & -135 & -4 & 133 & 133 & -2 & -137 & 4 & 8.6055 \\
\hline 39 & 198 & 216 & 6 & -213 & -213 & 3 & 219 & -6 & 8.7247 \\
\hline 40 & -187 & -205 & -6 & 202 & 202 & -3 & -208 & 6 & 8.9021 \\
\hline 41 & 134 & 146 & 4 & -144 & -144 & 2 & 148 & -4 & 9.0213 \\
\hline 42 & 70 & 76 & 2 & -75 & -75 & 1 & 77 & -2 & 9.318 \\
\hline 43 & 6 & 6 & 0 & -6 & -6 & 0 & 6 & 0 & 9.6146 \\
\hline 44 & -58 & -64 & -2 & 63 & 63 & -1 & -65 & 2 & 9.9113 \\
\hline 45 & -122 & -134 & -4 & 132 & 132 & -2 & -136 & 4 & 10.208 \\
\hline 46 & 199 & 217 & 6 & -214 & -214 & 3 & 220 & -6 & 10.327 \\
\hline 47 & -186 & -204 & -6 & 201 & 201 & -3 & -207 & 6 & 10.505 \\
\hline 48 & 135 & 147 & 4 & -145 & -145 & 2 & 149 & -4 & 10.624 \\
\hline
\end{tabular}




\section{Continued}

\begin{tabular}{|c|c|c|c|c|c|c|c|c|c|}
\hline 49 & 71 & 77 & 2 & -76 & -76 & 1 & 78 & -2 & 10.92 \\
\hline 50 & 7 & 7 & 0 & -7 & -7 & 0 & 7 & 0 & 11.217 \\
\hline 51 & -57 & -63 & -2 & 62 & 62 & -1 & -64 & 2 & 11.514 \\
\hline 52 & -121 & -133 & -4 & 131 & 131 & -2 & -135 & 4 & 11.81 \\
\hline 53 & 200 & 218 & 6 & -215 & -215 & 3 & 221 & -6 & 11.93 \\
\hline 54 & -185 & -203 & -6 & 200 & 200 & -3 & -206 & 6 & 12.107 \\
\hline 55 & 136 & 148 & 4 & -146 & -146 & 2 & 150 & -4 & 12.226 \\
\hline 56 & 72 & 78 & 2 & -77 & -77 & 1 & 79 & -2 & 12.523 \\
\hline 57 & 8 & 8 & 0 & -8 & -8 & 0 & 8 & 0 & 12.819 \\
\hline 58 & -56 & -62 & -2 & 61 & 61 & -1 & -63 & 2 & 13.116 \\
\hline 59 & -120 & -132 & -4 & 130 & 130 & -2 & -134 & 4 & 13.413 \\
\hline 60 & 201 & 219 & 6 & -216 & -216 & 3 & 222 & -6 & 13.532 \\
\hline 61 & -184 & -202 & -6 & 199 & 199 & -3 & -205 & 6 & 13.709 \\
\hline 62 & 137 & 149 & 4 & -147 & -147 & 2 & 151 & -4 & 13.829 \\
\hline 63 & 73 & 79 & 2 & -78 & -78 & 1 & 80 & -2 & 14.125 \\
\hline 64 & 9 & 9 & 0 & -9 & -9 & 0 & 9 & 0 & 14.422 \\
\hline 65 & -55 & -61 & -2 & 60 & 60 & -1 & -62 & 2 & 14.719 \\
\hline 66 & -119 & -131 & -4 & 129 & 129 & -2 & -133 & 4 & 15.015 \\
\hline 67 & 202 & 220 & 6 & -217 & -217 & 3 & 223 & -6 & 15.134 \\
\hline 68 & -183 & -201 & -6 & 198 & 198 & -3 & -204 & 6 & 15.312 \\
\hline 69 & 138 & 150 & 4 & -148 & -148 & 2 & 152 & -4 & 15.431 \\
\hline 70 & 74 & 80 & 2 & -79 & -79 & 1 & 81 & -2 & 15.728 \\
\hline 71 & 10 & 10 & 0 & -10 & -10 & 0 & 10 & 0 & 16.024 \\
\hline 72 & -54 & -60 & -2 & 59 & 59 & -1 & -61 & 2 & 16.321 \\
\hline 73 & -118 & -130 & -4 & 128 & 128 & -2 & -132 & 4 & 16.618 \\
\hline 74 & 203 & 221 & 6 & -218 & -218 & 3 & 224 & -6 & 16.737 \\
\hline 75 & -182 & -200 & -6 & 197 & 197 & -3 & -203 & 6 & 16.914 \\
\hline 76 & 139 & 151 & 4 & -149 & -149 & 2 & 153 & -4 & 17.034 \\
\hline 77 & 75 & 81 & 2 & -80 & -80 & 1 & 82 & -2 & 17.33 \\
\hline 78 & 11 & 11 & 0 & -11 & -11 & 0 & 11 & 0 & 17.627 \\
\hline 79 & -53 & -59 & -2 & 58 & 58 & -1 & -60 & 2 & 17.923 \\
\hline 80 & -117 & -129 & -4 & 127 & 127 & -2 & -131 & 4 & 18.22 \\
\hline 81 & 204 & 222 & 6 & -219 & -219 & 3 & 225 & -6 & 18.339 \\
\hline 82 & -181 & -199 & -6 & 196 & 196 & -3 & -202 & 6 & 18.517 \\
\hline 83 & 140 & 152 & 4 & -150 & -150 & 2 & 154 & -4 & 18.636 \\
\hline 84 & 76 & 82 & 2 & -81 & -81 & 1 & 83 & -2 & 18.933 \\
\hline 85 & 12 & 12 & 0 & -12 & -12 & 0 & 12 & 0 & 19.229 \\
\hline 86 & -52 & -58 & -2 & 57 & 57 & -1 & -59 & 2 & 19.526 \\
\hline 87 & -116 & -128 & -4 & 126 & 126 & -2 & -130 & 4 & 19.823 \\
\hline 88 & 205 & 223 & 6 & -220 & -220 & 3 & 226 & -6 & 19.942 \\
\hline 89 & -180 & -198 & -6 & 195 & 195 & -3 & -201 & 6 & 20.119 \\
\hline 90 & 141 & 153 & 4 & -151 & -151 & 2 & 155 & -4 & 20.238 \\
\hline 91 & 77 & 83 & 2 & -82 & -82 & 1 & 84 & -2 & 20.535 \\
\hline 92 & 13 & 13 & 0 & -13 & -13 & 0 & 13 & 0 & 20.832 \\
\hline 93 & -51 & -57 & -2 & 56 & 56 & -1 & -58 & 2 & 21.128 \\
\hline 94 & -115 & -127 & -4 & 125 & 125 & -2 & -129 & 4 & 21.425 \\
\hline 95 & 206 & 224 & 6 & -221 & -221 & 3 & 227 & -6 & 21.544 \\
\hline 96 & -179 & -197 & -6 & 194 & 194 & -3 & -200 & 6 & 21.722 \\
\hline 97 & 142 & 154 & 4 & -152 & -152 & 2 & 156 & -4 & 21.841 \\
\hline 98 & 78 & 84 & 2 & -83 & -83 & 1 & 85 & -2 & 22.137 \\
\hline
\end{tabular}




\section{Continued}

\begin{tabular}{|c|c|c|c|c|c|c|c|c|c|}
\hline 99 & 14 & 14 & 0 & -14 & -14 & 0 & 14 & 0 & 22.434 \\
\hline 100 & -50 & -56 & -2 & 55 & 55 & -1 & -57 & 2 & 22.731 \\
\hline 101 & -114 & -126 & -4 & 124 & 124 & -2 & -128 & 4 & 23.027 \\
\hline 102 & 207 & 225 & 6 & -222 & -222 & 3 & 228 & -6 & 23.147 \\
\hline 103 & -178 & -196 & -6 & 193 & 193 & -3 & -199 & 6 & 23.324 \\
\hline 104 & 143 & 155 & 4 & -153 & -153 & 2 & 157 & -4 & 23.443 \\
\hline 105 & 79 & 85 & 2 & -84 & -84 & 1 & 86 & -2 & 23.74 \\
\hline 106 & 15 & 15 & 0 & -15 & -15 & 0 & 15 & 0 & 24.037 \\
\hline 107 & -49 & -55 & -2 & 54 & 54 & -1 & -56 & 2 & 24.333 \\
\hline 108 & -113 & -125 & -4 & 123 & 123 & -2 & -127 & 4 & 24.63 \\
\hline 109 & 208 & 226 & 6 & -223 & -223 & 3 & 229 & -6 & 24.749 \\
\hline 110 & -177 & -195 & -6 & 192 & 192 & -3 & -198 & 6 & 24.926 \\
\hline 111 & 144 & 156 & 4 & -154 & -154 & 2 & 158 & -4 & 25.046 \\
\hline 112 & 80 & 86 & 2 & -85 & -85 & 1 & 87 & -2 & 25.342 \\
\hline 113 & 16 & 16 & 0 & -16 & -16 & 0 & 16 & 0 & 25.639 \\
\hline 114 & -48 & -54 & -2 & 53 & 53 & -1 & -55 & 2 & 25.936 \\
\hline 115 & -112 & -124 & -4 & 122 & 122 & -2 & -126 & 4 & 26.232 \\
\hline 116 & 209 & 227 & 6 & -224 & -224 & 3 & 230 & -6 & 26.351 \\
\hline 117 & -176 & -194 & -6 & 191 & 191 & -3 & -197 & 6 & 26.529 \\
\hline 118 & 145 & 157 & 4 & -155 & -155 & 2 & 159 & -4 & 26.648 \\
\hline 119 & 81 & 87 & 2 & -86 & -86 & 1 & 88 & -2 & 26.945 \\
\hline 120 & 17 & 17 & 0 & -17 & -17 & 0 & 17 & 0 & 27.241 \\
\hline 121 & -47 & -53 & -2 & 52 & 52 & -1 & -54 & 2 & 27.538 \\
\hline 122 & -111 & -123 & -4 & 121 & 121 & -2 & -125 & 4 & 27.835 \\
\hline 123 & 210 & 228 & 6 & -225 & -225 & 3 & 231 & -6 & 27.954 \\
\hline 124 & -175 & -193 & -6 & 190 & 190 & -3 & -196 & 6 & 28.131 \\
\hline 125 & 146 & 158 & 4 & -156 & -156 & 2 & 160 & -4 & 28.251 \\
\hline 126 & 82 & 88 & 2 & -87 & -87 & 1 & 89 & -2 & 28.547 \\
\hline 127 & 18 & 18 & 0 & -18 & -18 & 0 & 18 & 0 & 28.844 \\
\hline 128 & -46 & -52 & -2 & 51 & 51 & -1 & -53 & 2 & 29.14 \\
\hline 129 & -110 & -122 & -4 & 120 & 120 & -2 & -124 & 4 & 29.437 \\
\hline 130 & 211 & 229 & 6 & -226 & -226 & 3 & 232 & -6 & 29.556 \\
\hline 131 & -174 & -192 & -6 & 189 & 189 & -3 & -195 & 6 & 29.734 \\
\hline 132 & 147 & 159 & 4 & -157 & -157 & 2 & 161 & -4 & 29.853 \\
\hline 133 & 83 & 89 & 2 & -88 & -88 & 1 & 90 & -2 & 30.15 \\
\hline 134 & 19 & 19 & 0 & -19 & -19 & 0 & 19 & 0 & 30.446 \\
\hline 135 & -45 & -51 & -2 & 50 & 50 & -1 & -52 & 2 & 30.743 \\
\hline 136 & -109 & -121 & -4 & 119 & 119 & -2 & -123 & 4 & 31.04 \\
\hline 137 & 212 & 230 & 6 & -227 & -227 & 3 & 233 & -6 & 31.159 \\
\hline 138 & -173 & -191 & -6 & 188 & 188 & -3 & -194 & 6 & 31.336 \\
\hline 139 & 148 & 160 & 4 & -158 & -158 & 2 & 162 & -4 & 31.455 \\
\hline 140 & 84 & 90 & 2 & -89 & -89 & 1 & 91 & -2 & 31.752 \\
\hline 141 & 20 & 20 & 0 & -20 & -20 & 0 & 20 & 0 & 32.049 \\
\hline 142 & -44 & -50 & -2 & 49 & 49 & -1 & -51 & 2 & 32.345 \\
\hline 143 & -108 & -120 & -4 & 118 & 118 & -2 & -122 & 4 & 32.642 \\
\hline 144 & 213 & 231 & 6 & -228 & -228 & 3 & 234 & -6 & 32.761 \\
\hline 145 & -172 & -190 & -6 & 187 & 187 & -3 & -193 & 6 & 32.939 \\
\hline 146 & 149 & 161 & 4 & -159 & -159 & 2 & 163 & -4 & 33.058 \\
\hline 147 & 85 & 91 & 2 & -90 & -90 & 1 & 92 & -2 & 33.355 \\
\hline 148 & 21 & 21 & 0 & -21 & -21 & 0 & 21 & 0 & 33.651 \\
\hline 149 & -43 & -49 & -2 & 48 & 48 & -1 & -50 & 2 & 33.948 \\
\hline
\end{tabular}


Continued

\begin{tabular}{|c|c|c|c|c|c|c|c|c|c|}
\hline 150 & -107 & -119 & -4 & 117 & 117 & -2 & -121 & 4 & 34.244 \\
\hline 151 & 214 & 232 & 6 & -229 & -229 & 3 & 235 & -6 & 34.364 \\
\hline 152 & -171 & -189 & -6 & 186 & 186 & -3 & -192 & 6 & 34.541 \\
\hline 153 & 150 & 162 & 4 & -160 & -160 & 2 & 164 & -4 & 34.66 \\
\hline 154 & 86 & 92 & 2 & -91 & -91 & 1 & 93 & -2 & 34.957 \\
\hline 155 & 22 & 22 & 0 & -22 & -22 & 0 & 22 & 0 & 35.254 \\
\hline 156 & -42 & -48 & -2 & 47 & 47 & -1 & -49 & 2 & 35.55 \\
\hline 157 & -106 & -118 & -4 & 116 & 116 & -2 & -120 & 4 & 35.847 \\
\hline 158 & 215 & 233 & 6 & -230 & -230 & 3 & 236 & -6 & 35.966 \\
\hline 159 & -170 & -188 & -6 & 185 & 185 & -3 & -191 & 6 & 36.144 \\
\hline 160 & 151 & 163 & 4 & -161 & -161 & 2 & 165 & -4 & 36.263 \\
\hline 161 & 87 & 93 & 2 & -92 & -92 & 1 & 94 & -2 & 36.559 \\
\hline 162 & 23 & 23 & 0 & -23 & -23 & 0 & 23 & 0 & 36.856 \\
\hline 163 & -41 & -47 & -2 & 46 & 46 & -1 & -48 & 2 & 37.153 \\
\hline 164 & -105 & -117 & -4 & 115 & 115 & -2 & -119 & 4 & 37.449 \\
\hline 165 & 216 & 234 & 6 & -231 & -231 & 3 & 237 & -6 & 37.569 \\
\hline 166 & -169 & -187 & -6 & 184 & 184 & -3 & -190 & 6 & 37.746 \\
\hline 167 & 152 & 164 & 4 & -162 & -162 & 2 & 166 & -4 & 37.865 \\
\hline 168 & 88 & 94 & 2 & -93 & -93 & 1 & 95 & -2 & 38.162 \\
\hline 169 & 24 & 24 & 0 & -24 & -24 & 0 & 24 & 0 & 38.458 \\
\hline 170 & -40 & -46 & -2 & 45 & 45 & -1 & -47 & 2 & 38.755 \\
\hline 171 & -104 & -116 & -4 & 114 & 114 & -2 & -118 & 4 & 39.052 \\
\hline 172 & 217 & 235 & 6 & -232 & -232 & 3 & 238 & -6 & 39.171 \\
\hline 173 & -168 & -186 & -6 & 183 & 183 & -3 & -189 & 6 & 39.348 \\
\hline 174 & 153 & 165 & 4 & -163 & -163 & 2 & 167 & -4 & 39.468 \\
\hline 175 & 89 & 95 & 2 & -94 & -94 & 1 & 96 & -2 & 39.764 \\
\hline 176 & 25 & 25 & 0 & -25 & -25 & 0 & 25 & 0 & 40.061 \\
\hline 177 & -39 & -45 & -2 & 44 & 44 & -1 & -46 & 2 & 40.358 \\
\hline 178 & -103 & -115 & -4 & 113 & 113 & -2 & -117 & 4 & 40.654 \\
\hline 179 & 218 & 236 & 6 & -233 & -233 & 3 & 239 & -6 & 40.773 \\
\hline 180 & -167 & -185 & -6 & 182 & 182 & -3 & -188 & 6 & 40.951 \\
\hline 181 & 154 & 166 & 4 & -164 & -164 & 2 & 168 & -4 & 41.07 \\
\hline 182 & 90 & 96 & 2 & -95 & -95 & 1 & 97 & -2 & 41.367 \\
\hline 183 & 26 & 26 & 0 & -26 & -26 & 0 & 26 & 0 & 41.663 \\
\hline 184 & -38 & -44 & -2 & 43 & 43 & -1 & -45 & 2 & 41.96 \\
\hline 185 & -102 & -114 & -4 & 112 & 112 & -2 & -116 & 4 & 42.257 \\
\hline 186 & 219 & 237 & 6 & -234 & -234 & 3 & 240 & -6 & 42.376 \\
\hline 187 & -166 & -184 & -6 & 181 & 181 & -3 & -187 & 6 & 42.553 \\
\hline 188 & 155 & 167 & 4 & -165 & -165 & 2 & 169 & -4 & 42.672 \\
\hline 189 & 91 & 97 & 2 & -96 & -96 & 1 & 98 & -2 & 42.969 \\
\hline 190 & 27 & 27 & 0 & -27 & -27 & 0 & 27 & 0 & 43.266 \\
\hline 191 & -37 & -43 & -2 & 42 & 42 & -1 & -44 & 2 & 43.562 \\
\hline 192 & -101 & -113 & -4 & 111 & 111 & -2 & -115 & 4 & 43.859 \\
\hline 193 & 220 & 238 & 6 & -235 & -235 & 3 & 241 & -6 & 43.978 \\
\hline 194 & -165 & -183 & -6 & 180 & 180 & -3 & -186 & 6 & 44.156 \\
\hline 195 & 156 & 168 & 4 & -166 & -166 & 2 & 170 & -4 & 44.275 \\
\hline 196 & 92 & 98 & 2 & -97 & -97 & 1 & 99 & -2 & 44.572 \\
\hline 197 & 28 & 28 & 0 & -28 & -28 & 0 & 28 & 0 & 44.868 \\
\hline 198 & -36 & -42 & -2 & 41 & 41 & -1 & -43 & 2 & 45.165 \\
\hline 199 & -100 & -112 & -4 & 110 & 110 & -2 & -114 & 4 & 45.461 \\
\hline 200 & 221 & 239 & 6 & -236 & -236 & 3 & 242 & -6 & 45.581 \\
\hline
\end{tabular}


use dimensional analysis to check that Eq.1 through Eq.4 are unitless equations.

$$
\begin{aligned}
& \left(\frac{c^{6} F^{2}}{A^{2} G N R w}\right)^{\frac{1}{6}}=\left(\frac{49}{\pi^{2}}\right)^{9} \frac{\pi^{\frac{7}{12}} \times 5^{\frac{5}{2}}}{\zeta(3)^{2}} \\
& \left(\frac{F^{2} R^{5} w^{5}}{A^{2} G h^{6} N^{7}}\right)^{\frac{1}{6}}=\left(\frac{49}{\pi^{2}}\right)^{13} \frac{2^{\frac{2}{7}}}{5^{2} \pi^{\frac{7}{12}}} \\
& \left(\frac{c^{3} F^{2} R^{2} w^{2}}{A^{2} G h^{3} N^{4}}\right)^{\frac{1}{3}}=\left(\frac{49}{\pi^{2}}\right)^{22} \frac{2^{\frac{2}{7}} \times 5^{\frac{1}{2}}}{\zeta(3)^{2}} \\
& \left(\frac{c h N}{R w}\right)=\frac{\pi^{\frac{55}{6}} \times 5^{\frac{9}{2}}}{2^{\frac{2}{7}} \times 7^{8} \times \zeta(3)^{2}} \approx\left(\frac{49}{\pi^{2}}\right)
\end{aligned}
$$

Figure 1 is a plot of Table 1, and is intended to show that the system of equations in Table $\mathbf{1}$ is not random but very periodic. The green line represents the natural $\log$ of the right hand sides of the equations and the other lines represent the exponent powers of the physical constants.

Figure $\mathbf{2}$ is also a plot of Table 1, where the equations of the table have been resorted based on the values of the ninth column of the table, instead of the tenth column.
Figures 1 and 2 should prove that the family of unitless equations contained in Table $\mathbf{1}$ is not random but instead is a structure made up of periodic waveforms.

\section{DISCUSSION}

Once we know that the dimensionality of the left hand sides of the equations are correct, then our focus switches to the right hand side of the equations. One should note by definition all the physical constants on the left hand side are measurements and have limited accuracy.

Obviously the equations based physical measurements can not be more accurate than the measurements themselves. My method was to give the Maple software program the benefit of the doubt when computing the right hand sides of the equations.

For example while factoring and processing Eq.3 with Maple's identify command, the Apery's constant [9] appears in the result. Apery's constant can be expressed as a series, which means we could convert the right hand side of Eq.3 into a series just by redistributing some of the factors. For this reason I left Apery's constant in the answer which propagated to other the equations.

I view the form of the right hand sides of the equations as an idealized guess times an error term which was supplied by the reduced row echelon operation.

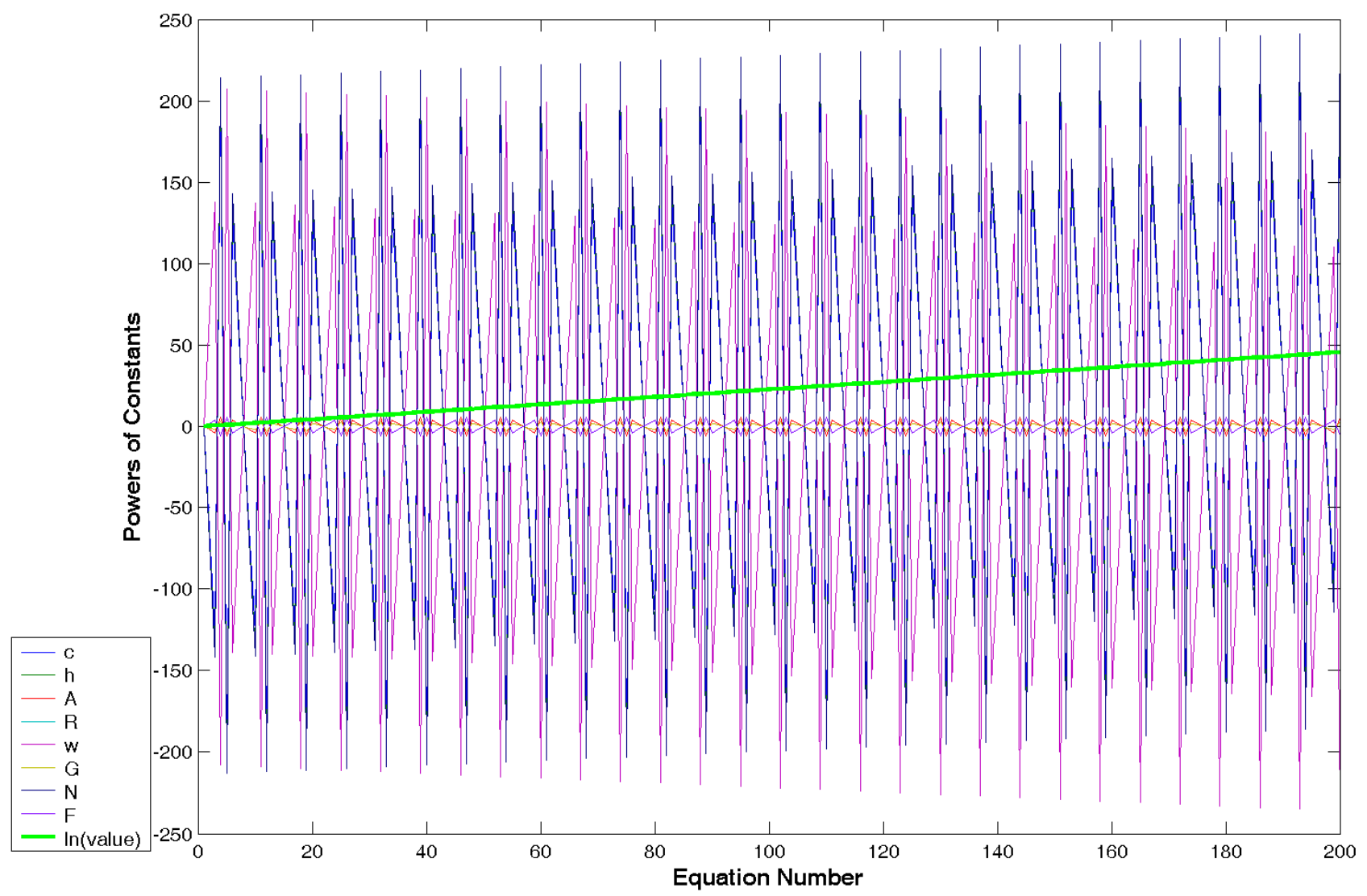

Figure 1. Plot of Table 1 sorted by the right hand side values. 


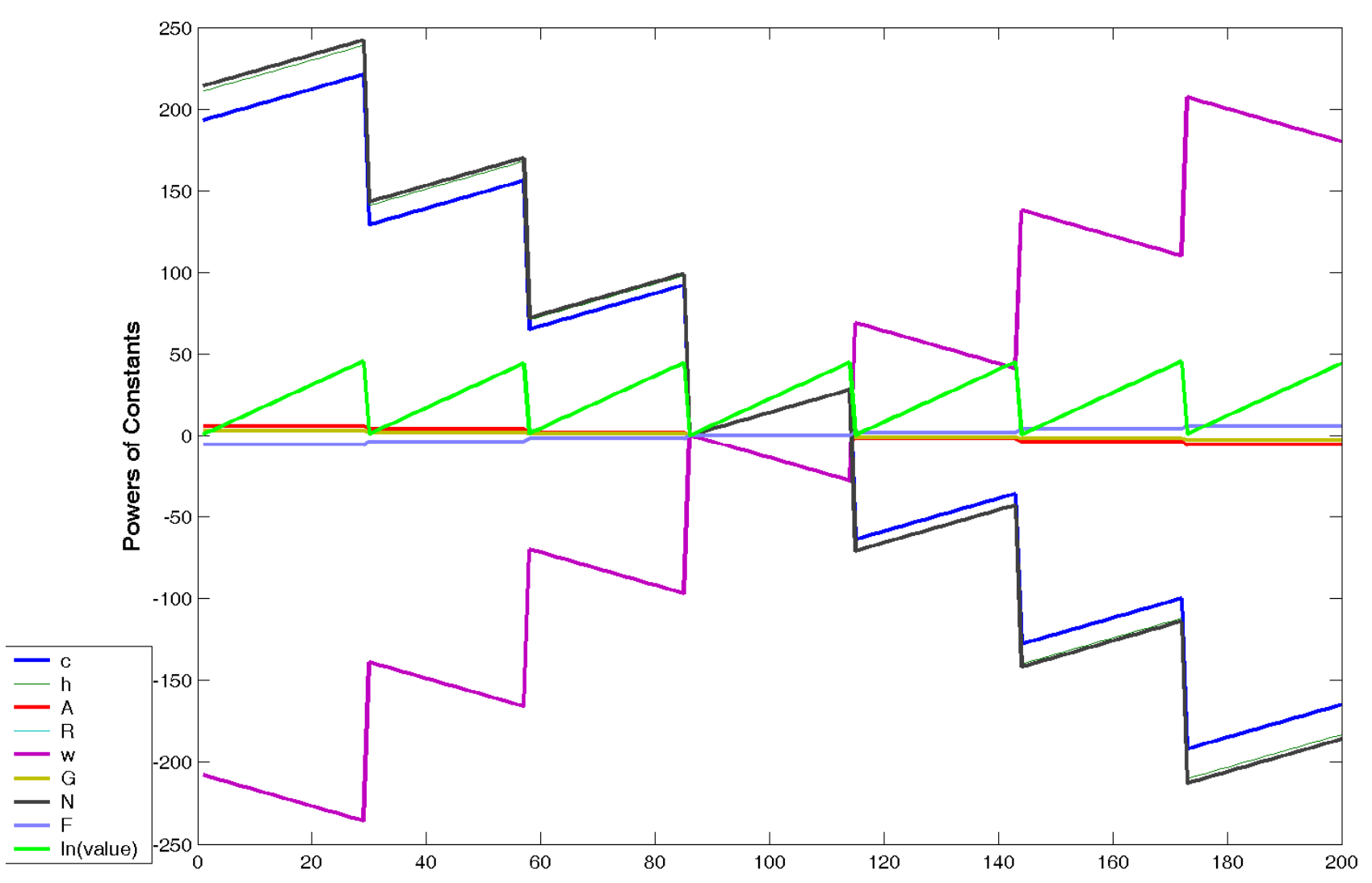

Figure 2. Plot of Table 1 sorted by the exponent values of the Faraday constant term.

A problem is that the right hand side of the equations are inherently more accurate than the left hand side of the equations; which means any exact answer found by my method is merely a good guess.

On the other hand, these guesses appear to have over seven significant digits of accuracy. Practically speaking, the right hand sides of Eq.1 through Eq.4 are close enough to the "right answers" to solve most problems and if one wishes more accuracy one can always use the left hand side to directly compute a decimal value.

\section{SUMMARY}

In some ways, this paper is mundane. We have a family of similar equations where any single equation can be proven with dimensional analysis to be unitless.

Assuming that a suitable expression can be found for the right hand sides of the equations, then most of these equations could be used like a Swiss army knife to change from one physical constant to another.

On the mundane side we basically have a relationship between nine constants that connects the constants like a key ring. On the other hand, one could argue that the relationships shown in this paper existed before any of the physical constants were measured.

Obviously I can not address the range of philosophical issues that this paper may cause. To answer the reader's unspoken question, I do not know why these relationships exist; I only know that each time that I check them they seem to be correct. I invite other papers to address the deeper issues and physical interpretations of my equations.

A database of over 17,000 equations is available for download; the reader is encouraged to download the database and verify my work. By definition the terms of these equations tend to be self canceling, meaning if you make the wrong substitution, the whole left hand side can disappear and just leave a number. This has happened to me quite a few times in the last few months, which leads me to my final statement of the paper: "I claim nothing."

\section{REFERENCES}

[1] Rømer, O. (1677) Lettre No 2104. In: J. Bosscha, Ed., Oeuvres Complètes de Christiaan Huygens, M. Nijhoff, La Haye, 1888-1950.

[2] Planck, M. (1920) The genesis and present state of development of the quantum theory. Nobel Lecture, Elsevier Publishing, Amsterdam.

[3] Feynman, R., Leighton, R. and Sands, M. (1989) The Feynman lectures on physics, vol. 1. Addison Wesley, Boston, 35-2-35-3.

[4] Avogadro, A. (1811) Essai d'une maniere de determiner 
les masses relatives des molecules elementaires des corps, et les proportions selon lesquelles elles entrent dans ces combinaisons. Journal de Physique, 73, 58-76.

[5] Newton, I. and Turnbull, H.W. (1960) The corresponddence of Isaac Newton: Volume 2 (1676-1687). Cambridge University Press, Cambridge, 309.

[6] Maxwell, J.C. (1904) Treatise on electricity and magnetism. Clarendon Press, Oxford, 173.

[7] Faraday, M. (1859) Experimental researches in chemistry and Physics. Richard Taylor and William Francis, Cambridge.

[8] Менделеев, Д.И. (1874) О сжимаемости газов (Из лаборатории С.-Петербургского Университета). Журнал русского химического общества и физического общества. Том 6, 309-352.

[9] Apéry, R. (1979) Irrationalité de $\zeta(2)$ et $\zeta(3)$. Astérisque, 61, 11-13. 


\section{APPENDIX I}

\#include "stdio.h"

\#define searchsize 29

// Search Size Should be an Odd Interger Greater than 5

// nvcc helloworld08g29.cu -o world08g29 -arch=sm_21 -maxrregcount $=24$-ccbin $=$ gcc- 4.4

// Note size 29 runs in about 15 seconds on a GTX560, size 79 runs in a few hours.

// All rights reserved, M. Snyder June 18, 2013

// Looking for solutions of $(\mathrm{x} 4-\mathrm{x} 5)^{\wedge} 2+(\mathrm{x} 3+\mathrm{x} 8)^{\wedge} 2+(\mathrm{x} 4+\mathrm{x} 7+\mathrm{x} 8)^{\wedge} 2+(\mathrm{x} 2+\mathrm{x} 4-\mathrm{x} 6)^{\wedge} 2+(-2$ $*(\mathrm{x} 4+\mathrm{x} 6)+\mathrm{x} 8-\mathrm{x} 1-\mathrm{x} 2)^{\wedge} 2+(2 *(\mathrm{x} 2+\mathrm{x} 4)+\mathrm{x} 1+\mathrm{x} 5+3 * \mathrm{x} 6)^{\wedge} 2=0$

$\{$ global_ void helloworld()

int $\mathrm{x} 1, \mathrm{x} 2, \mathrm{x} 3, \mathrm{x} 4, \mathrm{x} 5, \mathrm{x} 6, \mathrm{x} 7, \mathrm{x} 8$,rlow,rhgh;

rlow $=-((\operatorname{gridDim} . x-1) / 2)$;

rhgh $=(($ gridDim. $x-1) / 2)$;

$\mathrm{x} 1=$ blockIdx. $\mathrm{x}+$ rlow;

$\mathrm{x} 2=$ blockIdx.y+rlow;

$\mathrm{x} 3=$ threadIdx. $\mathrm{x}+$ rlow;

$\mathrm{x} 4=$ rlow;

$\mathrm{x} 5=$ rlow;

$\mathrm{x} 6=$ rlow;

$\mathrm{x} 7=$ rlow;

$\mathrm{x} 8=$ rlow;

while $(\mathrm{x} 8<$ rhgh $)$

\{

if $(\mathrm{x} 4+\mathrm{x} 7==-\mathrm{x} 8)\{$

if $(x 3=-x 8)\{$

if $((x 2+x 4)==x 6)\{$

if $(2 *(x 2+x 4)+x 1+x 5==-3 * x 6)\{$

if $(\mathrm{x} 4==\mathrm{x} 5)$

if $(-2 *(x 4+x 6)+x 8==x 1+x 2)\{$

printf(" \%+4d, $\%+4 d, \%+4 d, \%+4 d, \%+4 d, \%+4 d, \%+4 d, \%+$

$4 \mathrm{~d} \backslash n ", \mathrm{x} 1, \mathrm{x} 2, \mathrm{x} 3, \mathrm{x} 4, \mathrm{x} 5, \mathrm{x} 6, \mathrm{x} 7, \mathrm{x} 8$ );

\}

\}

\}

\}

\}

$\mathrm{x} 4=\mathrm{x} 4+1$;

if $(\mathrm{x} 4>$ rhgh $)\{\mathrm{x} 5=\mathrm{x} 5+1 ; \mathrm{x} 4=$ rlow;

if $(\mathrm{x} 5>$ rhgh) $\{x 6=x 6+1 ; \mathrm{x} 5=$ rlow;

if (x6>rhgh) $\{x 7=x 7+1 ; x 6=$ rlow;

if $(x 7>$ rhgh $)\{x 8=x 8+1 ; x 7=$ rlow;

\}
\}
\}
\}

int main()

\{

int rangeofsearch(searchsize),seconds;

$\operatorname{dim} 3$ grid,block;

grid. $x=$ rangeofsearch;

grid. $y=$ rangeofsearch;

block. $x=$ rangeofsearch;

size_t buf $=1 \mathrm{e} 7$;

cudaFuncSetCacheCon-

fig(helloworld,cudaFuncCachePreferL1);

cudaDeviceSetLimit(cudaLimitPrintfFifoSize, buf);

seconds $=$ time $($ NULL $)$;

helloworld $<<<$ grid,block $>>>($ );

cudaDeviceSynchronize();

printf ("In $\backslash n \backslash n C o m p l e t e$ CUDA Time: \%i ", int(time(NULL))-seconds);

return 0

\section{APPENDIX II}

A maple script one could use to check the program output.

with(ScientificConstants);

$\mathrm{c} 0:=\operatorname{evalf}($ Constant $(\mathrm{c}))$;

$\mathrm{c} 0 \mathrm{u}:=\operatorname{GetUnit}($ Constant(c));

$\mathrm{h} 0:=\operatorname{evalf(Constant(Planck}$ constant));

h0u := GetUnit(Constant(Planck_constant));

G0

evalf(Constant(Newtonian_constant_of_gravitation));

G0u $\quad:=\quad$ GetU-

nit(Constant(Newtonian_constant_of_gravitation));

w0

evalf(Constant(Wien_displacement_law_constant));

w0u := GetU-

nit(Constant(Wien_displacement_law_constant));

$\mathrm{R} 0$ := evalf(Constant(R));

$\mathrm{R} 0 \mathrm{u}:=\operatorname{GetUnit}(\operatorname{Constant}(\mathrm{R}))$;

$\mathrm{N} 0:=\operatorname{evalf}($ Constant(Avogadro constant));

NOu := GetUnit(Constant(Avogadro_constant));

A $0:=1$

A0u := Unit('A');

F0 := 96485.3399;

F0u := Unit('C')/Unit('mol'); 